\title{
From Spatiotemporal Curves to Reconstructed Depth
}

\author{
Rui Rodrigues \\ rpr@sim.di.uminho.pt \\ António Fernandes \\ Universidade do Minho, Portugal
}

Kees van Overveld

Fabian Ernst

overv@natlab.research.philips.com

fabian.ernst@philips.com

Philips Research, Eindhoven

\begin{abstract}
We present a novel approach for 3D reconstruction based on a set of images taken from a static scene. Our solution is inspired by the spatiotemporal analysis of video sequences. The method is based on a best fitting scheme for spatiotemporal curves, that allows us to compute 3D world coordinates of points within the scene. As opposed to a large number of current methods, our technique deals with random camera movements in a transparent way, and even performs better in these cases than with restrained motion such as pure translation. Robustness against occlusion and aliasing is inherent to the method as well.
\end{abstract}

Keywords: Depth Reconstruction; Spatiotemporal Video Analysis; Spatiotemporal Curves

\section{Introduction and Context}

Structure from Motion (SfM) is a field within computer vision still in progress. It deals with the problem of recovering the $3 \mathrm{D}$ structure of a scene from different perspective projections (e.g. video frames taken with a moving camera, or still images from a set of viewpoints) [1]. The range of applications of SfM techniques includes 3D-scene modelling, virtual view generation, $3 \mathrm{D} \mathrm{TV}$, image/video synthesis and autonomous navigation.

The availability of perspective projections with various projection centres allows us to estimate depth (the distance to the camera) of objects by comparing the projections' relative displacement of such objects in different frames, and using knowledge about camera position.

Several problems arise in this seemingly simple process. The first problem is related to camera information, which often is not readily available and has to be estimated as well. This is the camera calibration problem [20].

Second, it is not trivial to determine which parts of a set of digital images correspond to the same object or local feature in 3D space. This is known as the correspondence problem [3]. Some factors that contribute to the correspondence problem are image noise, periodic or absent textures and the occlusion of objects.

A third problem arises due to numerical or geometric instability: the stability problem.
The techniques described in the extensive literature available on SfM range from block matching algorithms to stochastic techniques, texture-based to feature-based. Many of the concepts are inherited from motion estimation research.

A large number of techniques analyse the case of consecutive frame pairs or triplets (e.g. trilinear tensor), estimating motion (depth) for each pair or triplet, and integrating the estimated data overtime as a post-processing operation. These techniques face stability problems in the fusion of the estimated data.

The reader is referred to [1] for an overview and references on SfM methods.

In this paper we focus on fusion, i.e. the seamless integration of information of all images simultaneously. The goal is to decrease significantly the stability problems mentioned previously.

First consider the particular case where the set of images comes from a video sequence. One way to perform this fusion is to regard video data as $3 \mathrm{D}$ information, time being the third dimension [4]. In this context, a sequence with a given number of frames can be represented as a colour distribution on the spatiotemporal domain, resulting in a spatiotemporal volume the VideoCube.

A video sequence possesses an interesting property not found in the general case of arbitrary sets of images: frame coherence. This is due to the fact that generally, camera parameters do not suffer from abrupt changes from one frame to the next. 
Consequently, there is a relatively small apparent motion between consecutive frames, reflected in a similarity between those frames that allows identifying spatiotemporal curves and surfaces, corresponding to the temporal path of objects throughout the scene.

The shape of these spatiotemporal entities is related to both the camera motion and the position of identifiable points in world space.

We propose a SfM method that estimates spatiotemporal curves (and therefore depth) for a set of interest points from the images. For each point a set of depth candidates is evaluated and ranked with a best-fit metric of the depth curves corresponding to those candidates.

We assume that camera parameters (both intrinsic and extrinsic) are known, or at least well estimated, and that the scene is static with little or no highlights.

The method presented here (and initially published in [2]), although inspired on the spatiotemporal analysis of video sequences, is general enough to be applied to arbitrary sets of images, as it does not critically depend on the spatiotemporal coherence of video sequences.

In the case of arbitrary sets of images, however, the notion of spatiotemporal curves assumes a definition of curves that also includes non-continuous discrete sequences of points.

Without loss of generality, we shall present our method within the VideoCube framework. This framework enhances the understanding of the underlying theoretical principles behind our method, since it is a simple and integrated concept showing the influence of varying depth for projected points over a set of images.

The main features of our approach are:

- The ability to deal with arbitrary collections of views, including smooth motion paths or discrete sets of views

- All frames can be used simultaneously.- The potential to benefit both from frame-to-frame coherence if available (meaning few occlusion differences on short time scale) and large baseline (meaning that due to the large total camera paths, geometric stability can be achieved).- The correspondence problem is tackled with a stochastically stable matching technique

- Robustness with respect to occlusion, noise and aliasing inherent to the method

The paper is organised as follows: The VideoCube is introduced in section 2, along with a brief overview of the literature in the area of video spatiotemporal analysis, followed by the concept of spatiotemporal curves in Section 3. In section 4 , a technique for estimating spatiotemporal curves is derived and later summarised in an algorithm. Section 5 contains the results obtained with a current implementation of the algorithm. Section 6 compares the technique with other SfM and spatiotemporal based techniques, showing the main differences. Finally, conclusions and future work close this paper in sections 7 and 8 , respectively.

\section{The VideoCube}

\subsection{Theoretical framework}

Assume that each point that belongs to an object is identified by its $3 \mathrm{D}$ co-ordinates and can be mapped to a colour. The set of all points $P_{l}$ with a colour defines the world $w$ :

$$
\mathbf{w}=\{<\mathbf{x}, \mathbf{y}, \mathbf{z}>\rightarrow \text { color } \mid \forall<\mathbf{x}, \mathbf{y}, \mathbf{z}>\in \text { OBJECTS }\}
$$

A perspective camera placed in this world can be described by the camera parameters $c p$

$\mathbf{c p}=\langle\mathbf{e}, \mathbf{h}, \mathbf{v}, \mathbf{k}, \mathbf{f}>$

where $e$ is the camera position, $h, v, k$ are the normal vectors (horizontal, vertical and looking direction) defining camera orientation and $f$ is the focal length of the camera.

Consider a finite plane $\pi$ lying at distance $f$ from the camera position $e$ and perpendicular to $k$ - the projection plane (Fig. 1.a). There is a subset of $w$ that can be projected on this plane.

The projection $P^{\prime}=<i, j>$ of a world point $P$ in $\pi$ is the intersection of the projection line $e P$ with $\pi$.

The depth of a point and its projection are defined as follows:

$$
\begin{aligned}
& \operatorname{Pdepth}(\mathrm{P}, \mathrm{cp})=\text { cp.k•(P-cp.e }) \\
& \operatorname{Pproj}(\mathbf{P}, \mathbf{c p})= \\
& <\text { cp.h॰(P - cp.e), cp.v•(P-cp.e) }>\text { * f / Pdepth(P, cp) }
\end{aligned}
$$

There is a large set of world points that project to $P^{\prime}$ (all the world points that lie on $e P$ ). Due to occlusion, only the point closest to the camera is registered in $\pi$.

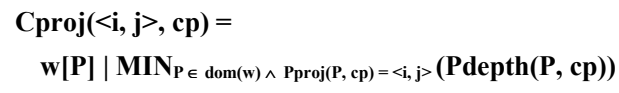

An image is therefore the set of world point colours projected in all its image points, considering occlusion and given a set of camera parameters:

$$
\operatorname{image}(\mathbf{c p})=\{<\mathbf{i}, \mathbf{j}>\rightarrow \operatorname{Cproj}(<\mathrm{i}, \mathrm{j}>, \mathbf{c p}) \mid \forall<\mathrm{i}, \mathrm{j}>\in \pi\}
$$

Consider now that the camera is moving, following a path $c p t$, associating a new set of camera parameters at each time instant when an image is recorded:

$$
\operatorname{cpt}[t]=\left\{t \rightarrow<e_{t}, h_{t}, v_{t}, k_{t}, f_{t}>\right\}
$$


Time may be either continuous or discrete. A video sequence is defined as the set of images obtained along the camera path:

$$
\mathbf{v s}(\operatorname{cpt})=\{\operatorname{image}(\operatorname{cpt}[\mathbf{t}]) \mid \forall t \in \operatorname{dom}(\operatorname{cpt})\}
$$

This video sequence can be seen as tri-dimensional data, namely bi-dimensional data varying along the time dimension. This leads to the VideoCube concept (Fig. 1.b): the spatiotemporal volume representing projected colour as a function of position in $\pi$ and time (see Fig. 2 for an example):

$$
\mathbf{v e}(\mathbf{c p t})=
$$

$\{<\mathbf{i}, \mathbf{j}, \mathbf{t}>\rightarrow \operatorname{Image}(\operatorname{cpt}[\mathbf{t}])[\mathbf{i}, \mathbf{j}] \mid \forall<\mathrm{i}, \mathrm{j}>\in \pi, \forall \mathbf{t} \in \operatorname{dom}(\operatorname{cpt})\}$

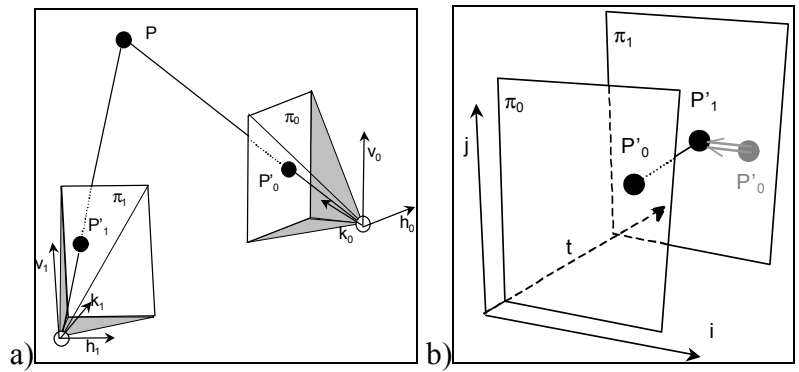

Fig. 1 Two frames of a sequence when viewed in: a) $3 D$-world space

b) Spatiotemporal space

\subsection{Previous VideoCube-related works}

One of the papers that first mentioned the VideoCube, and the "motion as orientation" effect of the paths formed in the $t$ direction, was done by Adelson and Bergen [4]. It is oriented to visual perception, and proposes to detect motion models based on energy and impulse response filters.

The works by Duc et al. [6], Wang et al. [7] and Moschenni et al. [8] are based on spatiotemporal analysis and are oriented to segmentation based on motion.

Otsuka et al. [10] try to benefit from the geometry of such volume by identifying "trajectory surfaces" (surfaces formed by edges and contours of images in spatiotemporal space) using Hough transforms [13]. The goal is to estimate the velocity component of the objects in a scene by determining the orientation of planes tangent to the detected surfaces.

Peng [11] slices the VideoCube in predefined orientations, and divides such slices into strips to detect line orientations that next are converted to optic flow.

Kim's work [9] on spatiotemporal analysis for edge detection and optical flow estimation gives an overview of the problems with spatiotemporal analysis. He mentions the problems due to noise and to time quantisation and the resulting lack of continuity inside the volume caused by that under-sampling and by image noise.
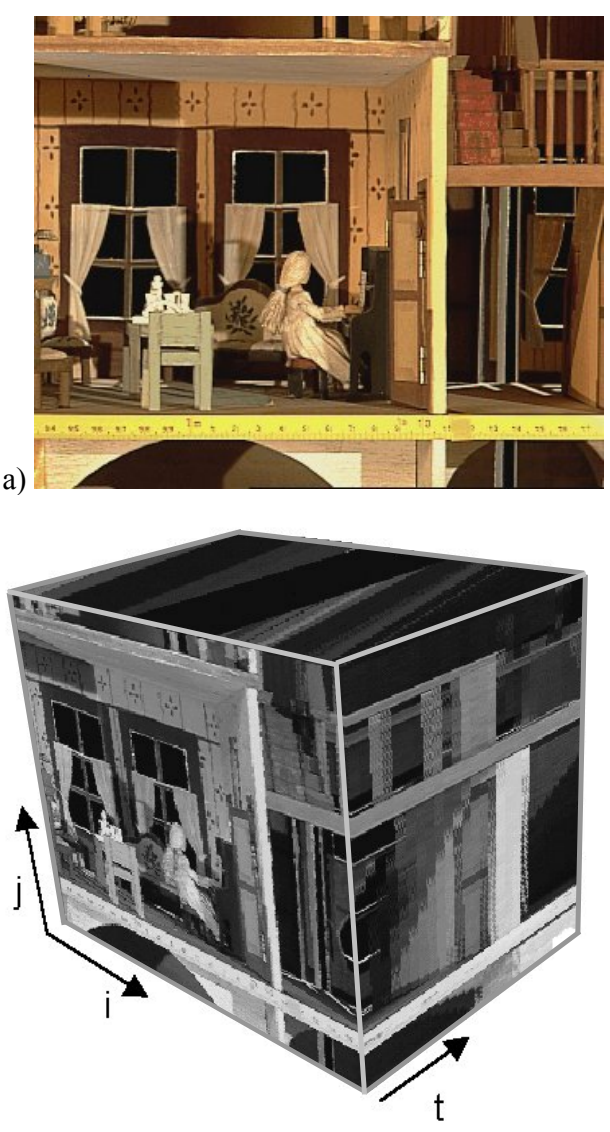

b)

Fig. 2 A VideoCube example

a) One frame of the "doll house" scene

b) The corresponding VideoCube

On a more probabilistic approach, Caplier and Luthon [5] extended Markov Random Fields (MRF) to the spatio-temporal model, defining a Markov Random Volume (which is an extension of concepts such as Markov chains and MRF).

These techniques share the fact that they are either restricted to simple camera motion models or small movements, and/or expensive to calculate.

Recently, Imiya and Kawamoto [12] proposed another Hough transform based approach. It uses a voting scheme to rate point correspondences over a series of frames, and to reconstruct world points. The authors randomly select a pair of points from the spatiotemporal data and check if they obey to the epipolar constraint. If so, a vote is accumulated to the corresponding world point. Reconstruction takes place by choosing the points that accumulated a larger number of votes. This method results in a high computational load, due to the large number of point pairs required. 


\section{Spatiotemporal Curves}

First consider the simple example of uniform (and slow) camera translation perpendicular to the camera's view direction. Assume that the camera moves in a horizontal world plane, as seen in the example of Fig. 2.

In the corresponding horizontal plane in the VideoCube, one can easily identify (nearly straight) line patterns (see Fig. 3 and Fig. 4). These lines are related to the relative apparent displacement of the objects' borders, due to camera motion.

The slope of these lines is a function of the distance of the objects to the camera. Lines that are nearly parallel to the time axis correspond to objects more distant (small apparent motion - large depth) and lines with sharper angles correspond to closer objects (large apparent motion - small depth).

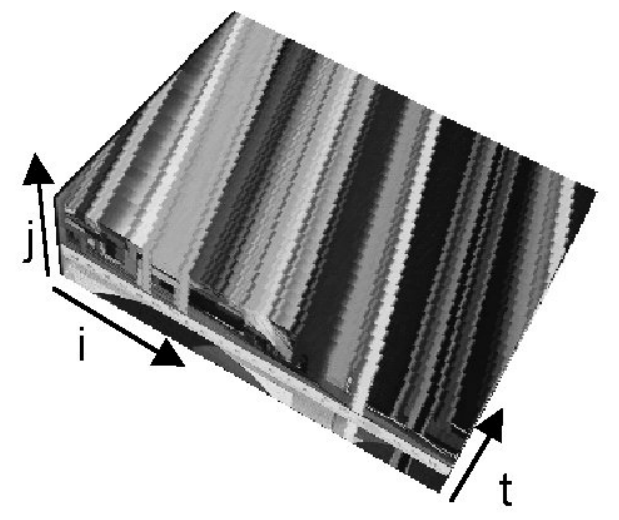

Fig. 3 A sliced VideoCube
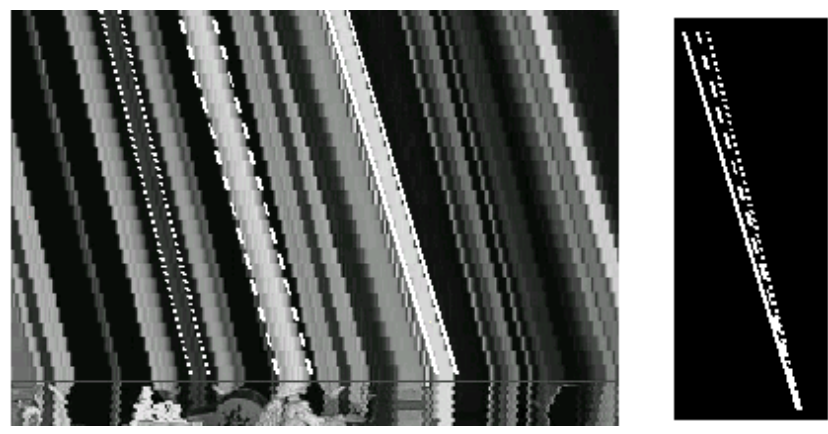

Fig. 4 Spatiotemporal lines and slope differences

Hence, if all camera parameters are known, depths can be estimated from the slopes of these lines, and a reconstruction is possible.

In this simple case, a properly parameterised Hough transform can detect the lines, and compute accurate estimates of the slopes (see [10] for an example).

Next, we consider a more complex camera motion model, such as a piecewise rotation or a superposition of rotation and translation. In this case, the lines of the previous example become more general and complex curves. The latter will be called spatiotemporal curves. Creating parameterised curve representations in this case is not straightforward. Even if such representation would be available, it would contain significantly more than two parameters, so a Hough transform would be unfeasible.

\section{Spatiotemporal Curve Estimation}

\subsection{Objective}

We aim to solve the following problem: given a VideoCube, in which spatiotemporal curves exist, obtain the set of 3D points that originated such curves.

\subsection{Pre-assumptions}

The scene must contain only static objects. This assures that any apparent motion in frames is only due to camera motion. For this same reason, lighting must also be constant, and there should be little or no highlights in the scene. Indeed, the position of a highlight varies with camera motion, yielding the same problem as with a moving object.

The method assumes that camera parameters such as focal length, trajectory, and orientation are well estimated.

On the other hand, we do not require:

- time continuity,

- any constraint on the camera path,

- limited baseline, nor

- constant occlusion relations

\subsection{Defining the Set of Interest Points (sip)}

Estimating depth for all points (and respective spatiotemporal curves) in the $v c$ is not feasible. Our approach reduces the data to be used in the estimation by considering only points that lie on contours in the individual images.

For this purpose, a transformation of the VideoCube is performed in order to extract contours from the images. These contours ideally should be as thin as possible. Further, it is desirable that they accurately represent the topology of the borders of the segments as they occur in the VideoCube. Therefore, the contour extraction algorithm is based on image segmentation.

The points to be used in the depth estimation are those which are present in a Contour Cube ( $c c)$, defined as:

$\mathbf{c c}=$

$\{<\mathbf{i}, \mathbf{j}, \mathbf{t}>\rightarrow \operatorname{contours}(\mathrm{vc}, \mathbf{i}, \mathbf{j}, \mathbf{t}) \mid \forall<\mathbf{i}, \mathbf{j}, \mathbf{t}>\in \operatorname{dom}(\mathrm{vc})\}$

where $v c$ is the original VideoCube. Points that are successfully detected as part of a contour are assigned TRUE; all other points are assigned FALSE. 
The set of interest points (sip) could therefore be defined as

$$
\operatorname{sip}=\left\{P^{\prime} \mid P^{\prime} \in \operatorname{dom}(c c) \wedge \operatorname{cc}\left[P^{\prime}\right]=\text { TRUE }\right\}
$$

A further step can be taken to reduce the number of interest points for which depth has to be estimated in order to get a meaningful 3D reconstruction. It is sufficient to estimate depth for corner and junction points on the contours. The depth values for the other points can then be interpolated, taking special care with the fact that junctions can represent more than one point, This is the case when a junction is due to occlusion.

Corner and junction points can be obtained either by applying feature detectors to the original images [22] or by extracting them directly from the contour information.

As we will require the contour information later for the depth estimation procedure, we have selected the second approach. This has the advantage of insuring that the chosen points are consistent with the contour information.

This process is depicted in Fig. 5. In Fig. 5.a) we see two segments in an original frame. The results of contour extraction are presented in Fig. 5.b). Next, junctions are extracted by means of a $3 \times 3$ template matching (Fig. 5.c). The parts connecting pairs of junctions may contain corners. These are detected by means a line generalisation (or simplification) algorithm, known in digital cartography literature [15]. The final result can be seen in Fig. 5.d). The set of interest points is the union of the junction points and corner points obtained in this way.

$$
\operatorname{sip}=\left\{P^{\prime} \mid P^{\prime} \in \operatorname{dom}(c c) \wedge P^{\prime} \in \text { corners_or_junctions(cc) }\right\} \text { (11) }
$$

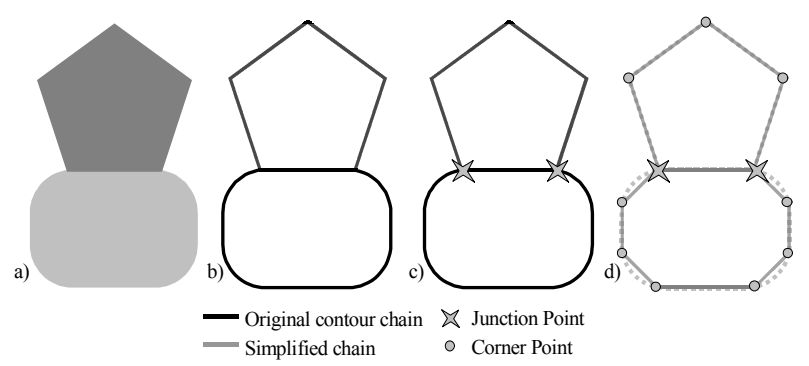

Fig. 5 Contour extraction and simplification

For the corner detection, we use the Douglas-Peucker [16] line simplification algorithm (also known as Ramer's [17] algorithm or the sandwich [18] algorithm). This algorithm has been identified as the most appropriate from both a mathematical [19] and a perceptual [15] point of view. It simplifies a chain of connected points by replacing it by a polyline. Initially, this polyline only connects the start and end points of the chain. Next, in order to give an increasingly better approximation to the shape of the original chain, intermediate points are added recursively.

In order not to miss any meaningful point, we use a conservative set of parameters. This increases the number of points found by the line simplification algorithm, but still reduces to a great length the original set of interest points. Furthermore, some extra non-feature points can be randomly selected to have some redundancy for accuracy increase.

There is no guarantee of correspondence between corners or junction points from distinct frames. Therefore, we keep the Contour Cube for the depth estimation procedure, described in the next section. Nevertheless, as mentioned before, depth is only directly estimated for points that are deemed as meaningful by the line simplification algorithm.

\subsection{Estimating depth}

Depth estimates can be obtained by trying to trace the spatiotemporal curves that exist on the VideoCube. However, the development of a tracing algorithm for spatiotemporal curves is not trivial due to both space and time aliasing.

We propose an alternative approach: to search depths for a given interest point $P^{\prime}$ by matching a set of candidate depth curves for $P^{\prime}$ against the implicit VideoCube spatiotemporal curves. A depth curve is defined as a spatiotemporal curve generated based on a candidate depth.

A depth curve can be generated in two steps. First, we define a reverse projection of a chosen spatiotemporal point $P$, of coordinates $<i r, j r, t r>$, with given camera parameters cpt $[t r]$ and an attributed depth $d$ as follows:

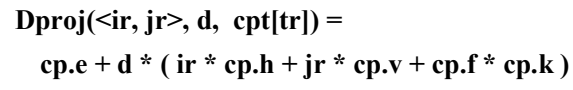

This reverse projection gives us a point in world coordinates. The corresponding depth curve stc is defined as:

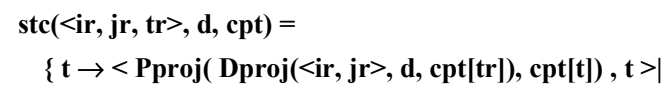

$\forall \mathbf{t} \in \operatorname{dom}(\mathbf{c p t})\}$

Assume a set of candidate depths $s c d$, from which we intend to pick the best one by means of a matching process that accounts to what extent the spatiotemporal curve associated with that depth can be identified in $c c$.

$$
\operatorname{scd}=\left\{\mathbf{d}_{0}, \mathbf{d}_{\mathbf{1}}, \ldots, \mathbf{d}_{\mathbf{n}} .\right\}
$$

The set of candidate depth curves $s c d c_{P}$, associated to $s c d$ is defined as:

$\operatorname{scdc}_{P}=\left\{\operatorname{stc}\left(P^{\prime}, \mathbf{d}, \mathbf{c p t}\right) \mid \mathbf{d} \in \operatorname{scd}\right\}$ 
The real spatiotemporal curve $r s c$ that contains $P^{\prime}$ in a particular frame is unknown. Let $\mathrm{P}$ be the world point that projects as $\mathrm{P}^{\prime}$ in that particular frame.

We shall consider the three possible cases for $\mathrm{P}$ :

a) $P$ has a projection in all frames and its projections are all identified as contour points.

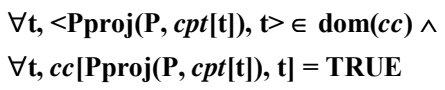

b) $P$ has a projection in all frames but in at least one frame it is not identified as a contour. This can be due to the occlusion of $P$, aliasing problems, faulty edge detection, or noise in the original VideoCube.

$$
\begin{aligned}
& \forall \mathrm{t},<\operatorname{Pproj}(\mathbf{P}, c p t[\mathbf{t}]), \mathbf{t}>\in \operatorname{dom}(c c) \wedge \\
& \exists \mathbf{t}: c c[\operatorname{Pproj}(\mathbf{P}, c p t[\mathbf{t}]), \mathbf{t}]=\mathbf{F A L S E}
\end{aligned}
$$

c) The projection of $P$ lies outside $c c$ in at least one frame.

$$
\exists \mathrm{t}:<\operatorname{Pproj}(\mathrm{P}, \operatorname{cpt}[\mathrm{t}]), \mathrm{t}>\notin \operatorname{dom}(c c)
$$

Let $P^{\prime}=<$ iref, jref, tref $>$ be a point projection existing in a reference frame tref, and $d c \in s c d c_{P}$. The depth curve $d c$ includes $P^{\prime}$ and is built based on a given depth estimate $d_{e}$ for $P^{\prime}$.

In the ideal case a), rsc intersects a contour in all frames in $c c$. If the depth estimate for $P^{\prime}$ in the reference frame is correct, that is, if the appropriate depth from $s c d$ has been chosen, $d c$ will also intersect a contour in all frames, i.e.:

$$
\forall t, \operatorname{cc}[\mathbf{d c}[\mathbf{t}]]=\mathbf{T R U E}
$$

where $d c[t]$ is the point intersection of $d c$ at frame $t$.

However, the most common situation is b). In this case, the number of contours that are actually intersected by $r s c$ may not be significant. Therefore we propose to select the best depth for $P^{\prime}$ from $s d c$ by minimizing a MatchError. This is defined as a function of the distance between the points of $d c$ and the contours in $c c$. A distance transform [14] of $c c$ is used to provide the required distances.

$$
\begin{aligned}
& \operatorname{dmc}(\mathbf{c c})= \\
& \{<\mathbf{i}, \mathbf{j}, \mathrm{t}>\rightarrow \operatorname{distanceToContour}(\mathrm{cc},[\mathrm{i}, \mathrm{j}, \mathrm{t}]) \mid
\end{aligned}
$$

$$
\forall<\mathbf{i}, \mathbf{j}, \mathbf{t}>\in \operatorname{dom}(\mathbf{c c})\}
$$

$$
\operatorname{MatchError}(\mathbf{s t c}, \mathbf{d m c})=\sum_{t} \mathbf{d t v c}[\operatorname{stc}[t], t]
$$

The metric MatchError will have a theoretical minimum when $d_{e}$ matches the real depth $d_{r}$. We refer to it as "theoretical" because, due to aliasing problems and noise, the depth corresponding to the actual minimum may be different from $d_{r}$. We shall come back to this issue later, for now we will accept the theoretical minimum as the real minimum.

The problem of depth curve estimation for a point $P^{\prime} \in \operatorname{sip}$, where $P^{\prime}$ belongs to a frame $t$ can now be stated as:

$$
\operatorname{MIN}_{\mathrm{d}} \text { MatchError( } \operatorname{stc}(<\mathrm{P} \text { ', }>\text {, d, cpt), dme ) }
$$

A search in depth space can be performed in order to find the minimum of MatchError. Using this approach, even with aliasing and noise, we can expect to find a depth $d$ with a MatchError value fairly close to zero. However, if this error is very high, above a predefined error threshold et, there is no confidence in the estimate, and therefore the point should be discarded from the final reconstruction. The minimisation process currently uses the Brent minimisation algorithm as described in [21], so that for each point, the initial $s d c$ is extended with new depth guesses provided by the algorithm.

However, degenerate cases may occur. To illustrate these, we will assume that we have a VideoCube generated from a camera translating horizontally, and this videocube is sliced by a horizontal plane as in the example from Section 3.

The first example of such a situation occurs when an estimated depth curve intersects several distinct real spatiotemporal curves, corresponding to unrelated contours in different frames (Fig. 6), providing a misleading match to a wrong depth candidate. This is called the "cross-matching" problem.

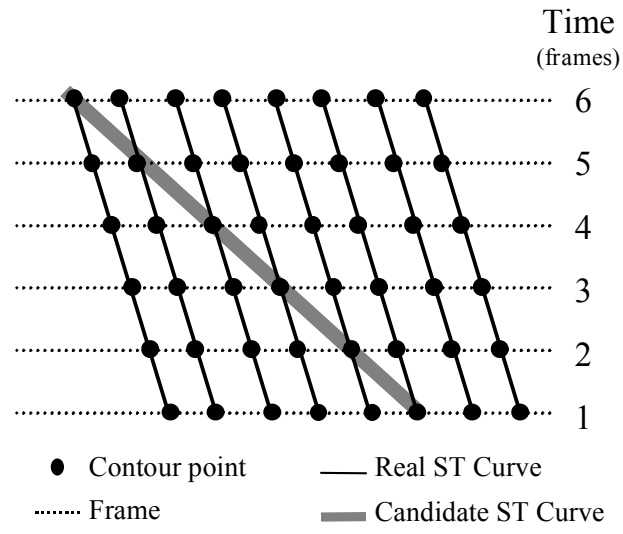

Fig. 6 Contour Cube "slice" representing the "cross-matching" phenomena

This yields a low (yet misleading) error for the corresponding depth, and consequently a low local minimum in MatchError as a function of depth. As a result, multiple wellseparated local minima below a minimum threshold may occur: one for the correct depth and several originated by "crossmatching" (Fig. 7). 


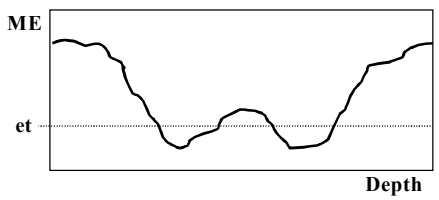

Fig. 7 Representation of MatchError vs. Depth when "crossmatching" occurs

In this case, we have a number of clearly distinct depth approved candidates for a point. Points close to the original point can be tested, and if the multiple local minima situation persists then the point should be dismissed as a contribution to the final 3D reconstruction. The use of regularisation can also be of assistance in these cases.

A particular example of this degeneration is when some contours are aligned with the direction of the camera movement. In such a situation the estimated depth curves for a given depth interval will intersect these contours (Fig. 8).

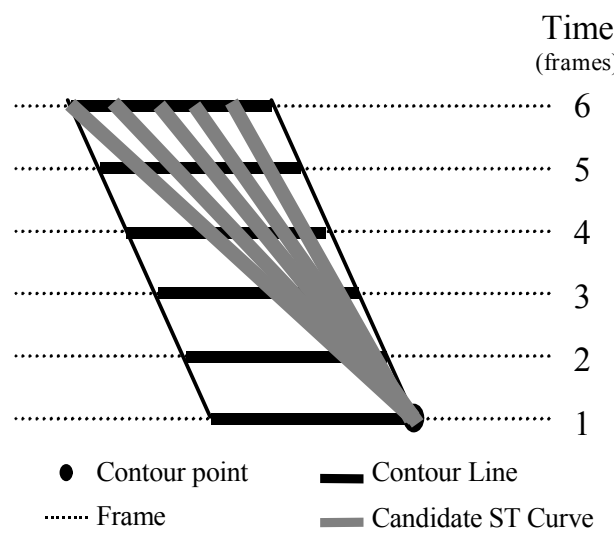

Fig. 8 Contour Cube "slice" representing the "cross-matching" phenomena for contours aligned with the camera path

This yields an interval of depths with low error: a "plateau" (Fig. 9).

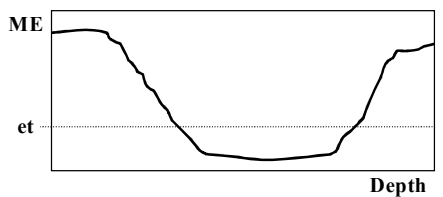

Fig. 9 Representation of MatchError vs. Depth when contours aligned with the camera path. The error "plateau" is easily identifiable.

Random, or irregular, camera movements will eliminate these situations in the general case. In general, irregular camera motion allows for more information about the scene to be extracted as opposed to simple translations or rotations of the camera. The method deals the same way with regular and irregular camera movements, without any special precaution
Let us consider now what happens if occlusion occurs for a set of frames. As defined in Eq. 15, if a point is occluded in a set of frames, the corresponding spatiotemporal curve is not present in those frames. This means that the estimated depth curve, which does not consider occlusion, will never be a perfect match for the spatiotemporal curve, as it will not intersect contours in the occluded frames in the general case (Fig. 10).

In such cases, the search is unlikely to achieve MatchError values below the error threshold for any candidate depth value. In the case of video sequences, an analysis of the individual frame errors for each depth should be performed to look for time segments of error values consistently above and below a frame error threshold eft (Fig. 11). If such sequences do exist then we can infer that the point is indeed occluded, and sequences of frame errors above the frame error threshold should be dismissed. A minimum length for sequences with frame error values below the threshold is required in order to have confidence in the estimate.

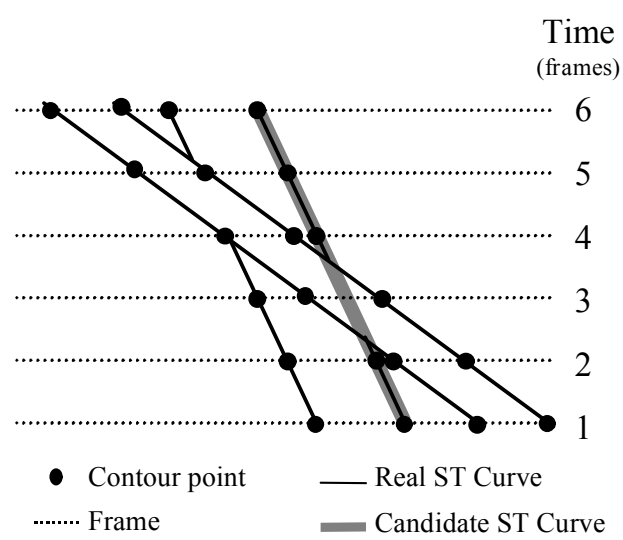

Fig. 10 Contour Cube "slice" representing an occlusion situation

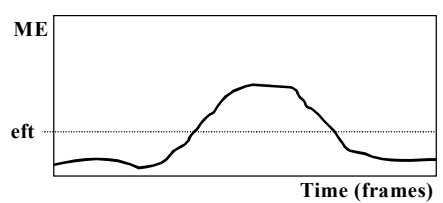

Fig. 11 Representation of MatchError vs. Time (frames) for a given depth candidate when occlusion occurs

In the general case of an arbitrary set of images, this reasoning does not apply. For this latter case a possible solution lies in regularisation.

Another degenerate situation occurs when the depth curve estimate lies outside the VideoCube in some frames (Fig. 12). Since no information is available in these frames it does not make sense to compute a MatchError for them (Fig. 13). 


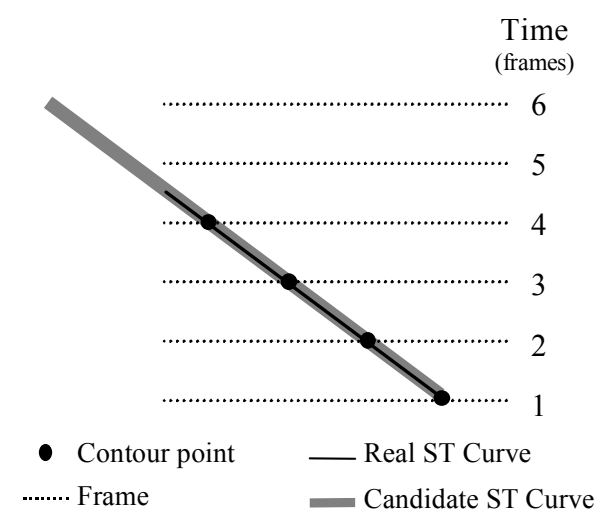

Fig. 12 Contour Cube "slice" representing an "out-of-sight" situation

If the number of frames that can be used to compute MatchError is below a threshold, then the resulting value should be given a low level of confidence.

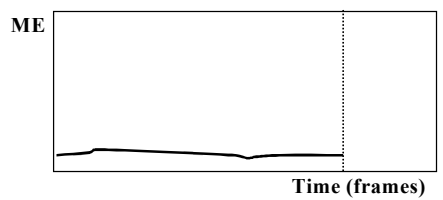

Fig. 13 Representation of MatchError vs. Time (frames) for a given depth candidate when an "out-of-sight" situation occurs

\subsection{The Algorithm}

An algorithm that applies the concepts discussed so far to the reconstruction of video scenes is now outlined.

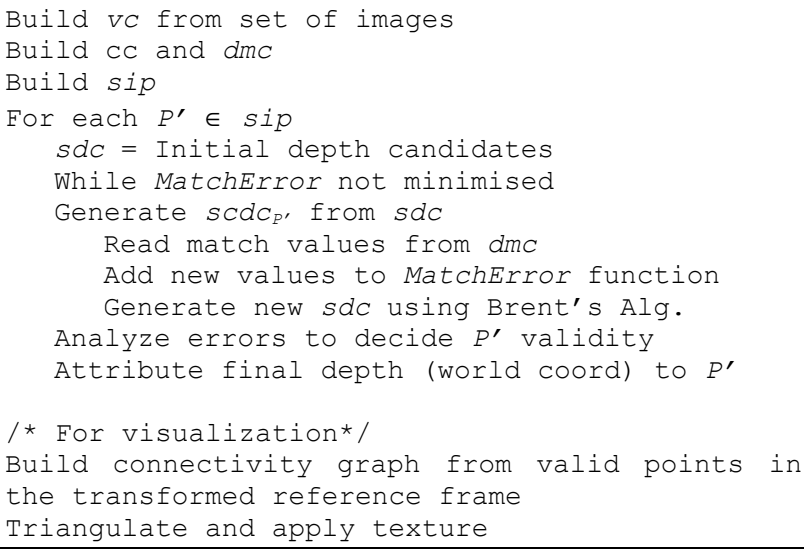

\section{Results}

In this section, we present some results obtained with the current implementation of the algorithm.

Sub-section 5.1 uses a simple synthetic scene to illustrate situations where occlusion and depth curves extending outside the VideoCube exist. Sub-sections 5.2 and 5.3 present the reconstruction of a synthetic and a real scene, respectively.

\subsection{Occlusion and Extra-VideoCube ST curves}

For this section, we built a synthetic scene consisting of a camera rotating $180^{\circ}$ around a set of three objects (Fig. 14) - a torus and two thin blocks.

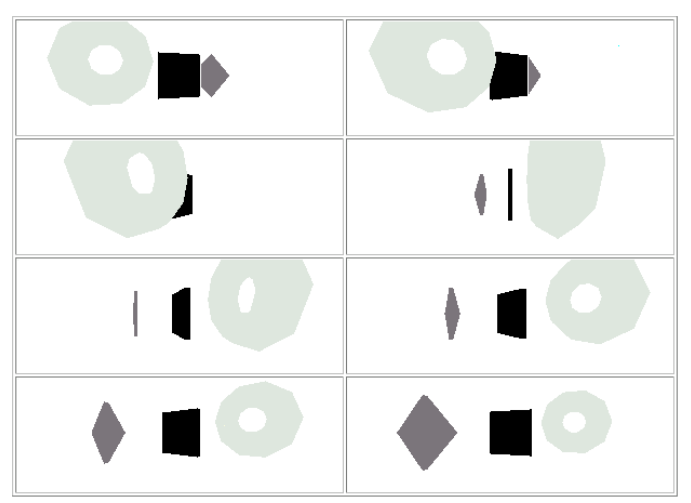

Fig. 14 Some frames of the sequence

The $c c$ corresponding to this sequence can be seen in Fig. 15, where the thick contours correspond to the TRUE values. A sip was chosen from the points of the first (left) frame, and the lines crossing $\mathrm{cc}$ are the depth curves corresponding to the selected depth for each point in sip.

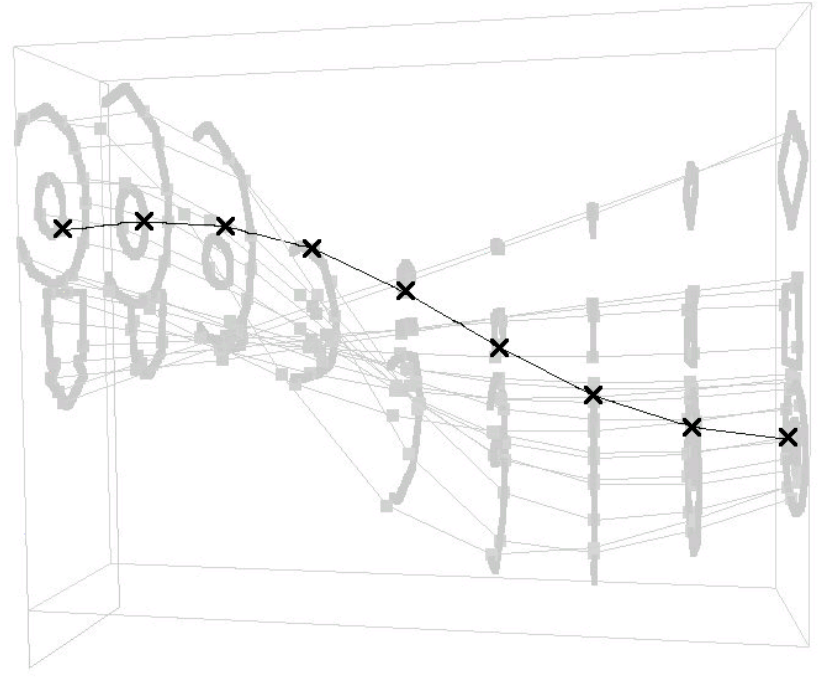

Fig. 15 A representation of a cc corresponding to 9 frames (the thick contours correspond to the TRUE values) and estimated depth curves for a sip

Notice the outlier depth curve (darkest line) extending from the inner circle of the torus. This is an example of a depth curve that, although it doesn't match any real spatiotemporal curve, intersects all contours by coincidence. Notice also how 
most points in the two blocks have good depth curves, despite being occluded by the torus on the frames corresponding to the centre of the VideoCube. Fig. 16.a) and Fig. 16.b) illustrate a $s c d c$ for a single point, and Fig. 16.c) and Fig. 16.d) show the selected depth curve.

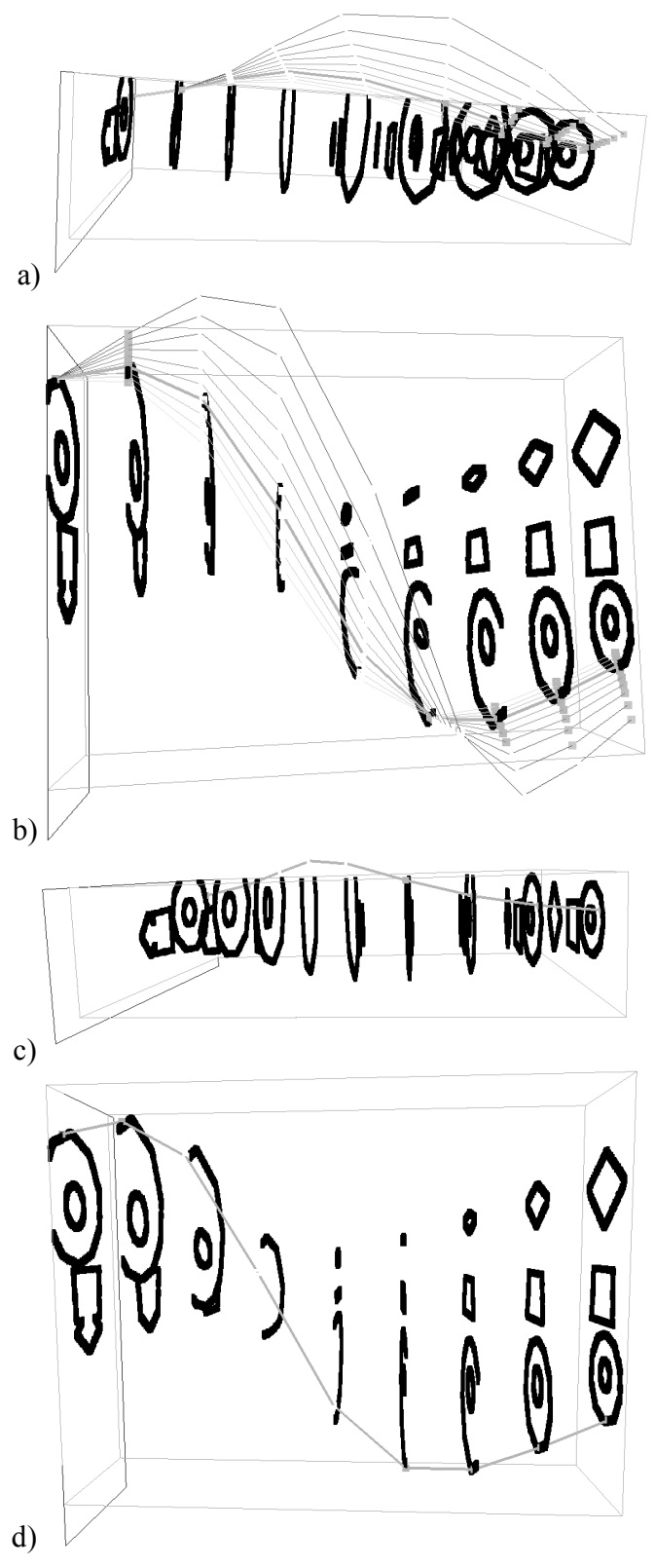

Fig. 16 Side (a) and top (b) views of scdc for a point and Side (c) and top (d) views of the selected $\mathrm{dc}$

One can see that the point is well traced outside the VideoCube, although the section of the $d c$ outside the VideoCube is not used in the matching. This occurs because there is enough evidence in the remaining frames to get a good estimate.

\subsection{Synthetic scene}

This scene is made up of 20 coloured boxes, arranged in a circle. The camera moved with random changes within a small interval in translation, rotation angle and axis. The original scene can be seen in Fig. 17. The camera positions are represented by small dots (bottom left of figure). The small line segments extending from each dot represent the camera's orientation, and the line connecting them is the camera path.
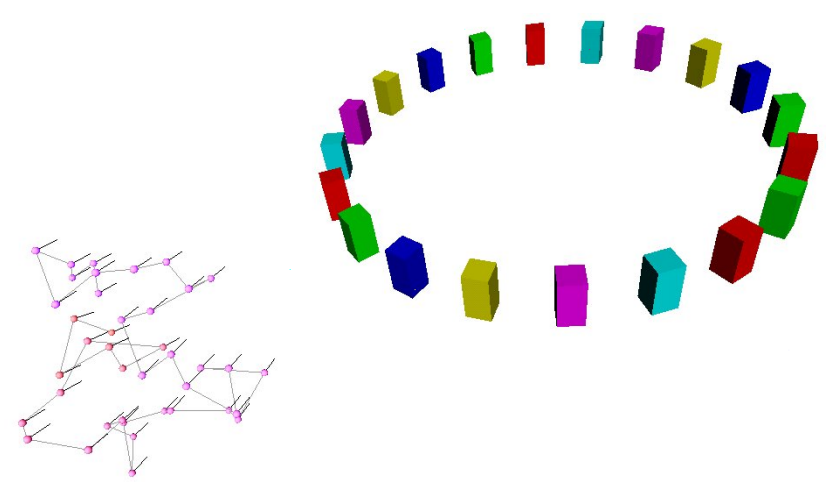

Fig. 17 The synthetic scene, including the camera positions

Fig. 18 shows three frames from the 40 used for the reconstruction.

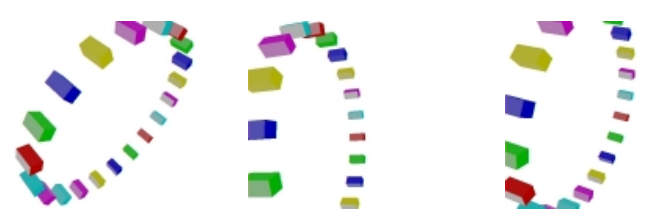

Fig. 18 Three frames of the synthetic scene

A sip of 400 points was processed, from which 279 where considered valid. Fig. 19 shows the valid reconstructed points (black) superimposed on the ground truth data (grey).

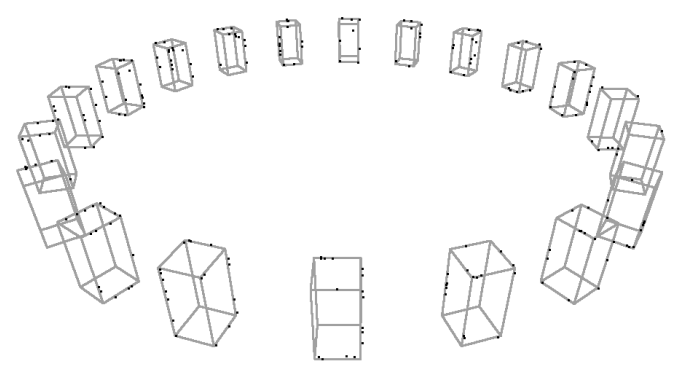

Fig. 19 Point reconstruction vs. ground truth

The final reconstruction, after triangulation and texturing, can be seen in Fig. 20. 


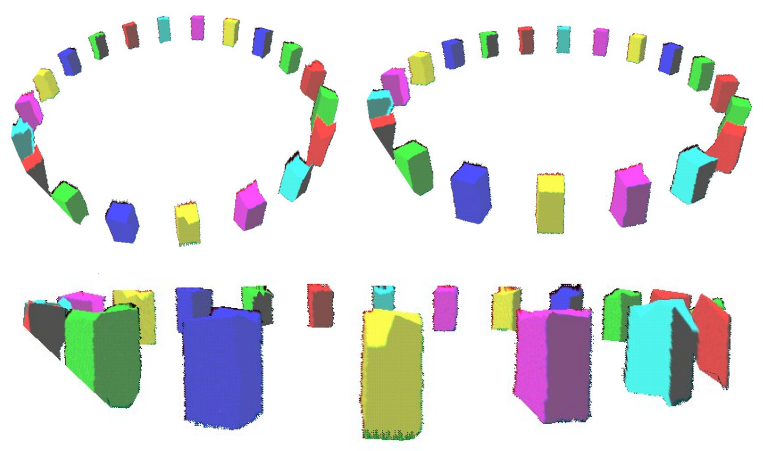

Fig. 20 Three views of the reconstruction

The reconstructed boxes lie in a circle as in the original frame. These results show good localisation of objects given the high number of occlusion occurrences and the number of points and frames used. The visible artefacts are the consequence of the reduced number of points used, which are insufficient for an accurate reconstruction.

\subsection{Real scene}

Due to restrictions on the available camera calibration methods, this sequence was recorded in a single camera movement, composed of a horizontal translation from left to right, and a vertical translation from top to bottom. It contains three objects, as can be seen in Fig. 21, where a subset of the 40 frames used is shown.

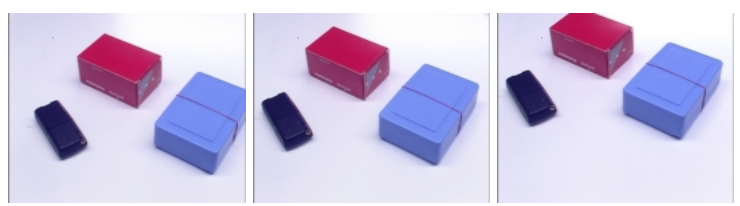

Fig. 21 Three frames of the real scene

In the scene reconstruction of Fig. 22, a sip of 300 points was built, from which 155 where considered valid.

a)

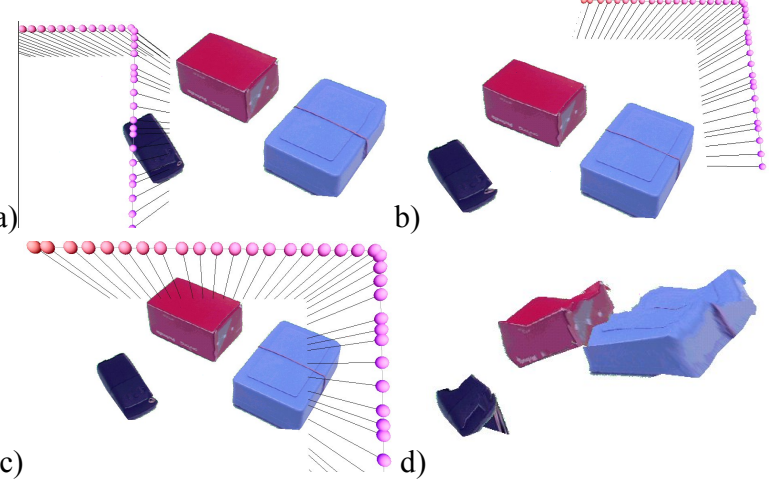

Fig. 22 Four views of the reconstructed scene

Fig. 22, a), b) and c) show the reconstruction from viewpoints close but outside the original camera path. In these three cases, the reconstruction preserves the shape and texture of the original.

In d) a view from a position and orientation radically different from the original cameras was used. Although some distorting artefacts can be seen, the results are still good in terms of localisation. If we take into account the fact that no regularisation is in use, the results obtained so far seem promising.

\section{Note on relations with other SfM approaches}

The distance-based match error is comparable to the match penalty or error measure of block matching techniques. However, with depth curves, this error is transparently accumulated over a pre-defined number of frames, and assigned directly to one single depth. In block/region matching techniques, the integration is usually done on a per block/region basis. Therefore, varying apparent motion of the same block on different frame pairs leads to matching penalties than are not easily correlated between each other.

Our method does not rely on simple motion models of camera translation or rotation, as opposed to some of the classical SfM methods, or spatiotemporal methods, as the ones presented in section 2. The assumption of irregular camera motion on our spatiotemporal framework is one of its strong points. In fact, as seen in the previous sections, an irregular motion of the camera can disambiguate some situations where scene objects would be aligned with a regular camera motion. Furthermore, the technique does not rely on either small or smooth camera motion between successive frames.

Implicit constraints to reduce the search space found on other matching techniques, such as epipolar geometry [20], also have their dual on the surface swept by the possible depth curves of a given point. If a point is projected on a given frame, its projections on the other frames have to lie on a spatiotemporal surface shaped by all the possible depth curves.

Regarding Imiya and Kawamoto's work, our approach differs in the fact that we consider an entire camera path and apply the votes to the entire depth curve. One advantage of using entire curves as matching entities is that all information over the entire time interval is taken into account: depth assignments are not necessarily based on frame-to-frame similarities. This gives potentially a better stability. In addition, our method requires a much smaller number of samples to be used, when compared to the point pairs needed for their random sampling. This means that the computational load of our method is substantially lower.

Finally, classical point tracking methods have the disadvantage of losing track of points when they are occluded for some frames. They are able to track them later again, but usually not able to link the two tracks. In addition, although they do not require special camera motion, they require small 
steps between adjacent camera positions. Our method overcomes both of these problems transparently.

\section{Conclusions}

A new technique for depth reconstruction from sets of images inspired on spatiotemporal analysis has been presented. It converts the depth estimation problem into the problem of matching spatiotemporal curves with the contours of a Contour Cube. A distance-based rating scheme is used for ranking the match quality, which implicitly reduces both the temporal and spatial aliasing problem.

It is assumed that the recorded scene is static and has constant lighting. Camera motion should be known but, as opposed to other techniques, it is not required to be regular. In fact, irregular camera movements improve the results in some situations, when compared to regular movements (such as translation), as it removes some of the ambiguities likely to arise from contours aligned with the camera path.

The integrating nature of the algorithm provides inherent robustness to occlusion. The results show that even without regularisation, depth estimations with consistent localisation and reconstruction can be achieved.

\section{Future Work}

It is known that regularisation plays an important role in 3D reconstruction. It is expected that a careful implementation of the regularisation will improve this method as well. Such implementation should remove outliers resulting from the degenerate cases presented in 4.4 , while preserving meaningful depth discontinuities, as the ones between objects in the foreground and in the background.

The method so far relies on geometric information taken from edge data. This is an advantage in terms of simplicity of processing, but a disadvantage, as it suffers from crossmatching. Photometric (texture) similarity could be used for confirmation or disambiguation of estimated depths.

Regarding occlusion, more sophisticated methods to deal with this problem are being studied. One possible process would be to iteratively re-estimate depth for points of low confidence by testing occlusion hypotheses. This could further improve the overall reconstruction. Another approach is based on frame coherence when considering video sequences.

Other common problems such as noise in the source video data, inaccurate camera calibration and sensitivity of edge detection algorithms also require further testing.

In the case of video sequences, the spatiotemporal curves and surfaces can be explored in several directions, such as its usage for camera calibration and video coding.

\section{Acknowledgements}

The work presented here is supported by Grant PRAXIS XXI/BD/20322/99, sponsored by Fundação para a Ciência e Tecnologia (the Portuguese Foundation for Science and Technology).

\section{References}

[1] T. Jebara, A. Azarbayejani and A. Pentland. 3D structure from 2D motion. IEEE Signal Processing Magazine, 16(3), 1999.

[2] R. Rodrigues, A. Fernandes, K. van Overveld, and F. Ernst. Reconstructing Depth from Spatiotemporal Curves, Vision Interface 2002,Proceedings, pages 252-259, Canada, May 2002.

[3] A. Redert, E. Hendriks, and J. Biemond. Correspondence estimation in image pairs. IEEE Signal Processing Magazine, 16(3):29-46, 1999.

[4] E. H. Adelson and J. R. Bergen. Spatiotemporal energy models for the perception of motion. J. of the Optical Society of America A, 2(2):284-299, 1985.

[5] A. Caplier and F. Luthon. A new spatiotemporal approach for image analysis application to motion detection. Lecture Notes in Computer Science, 970:246-253, 1995.

[6] B. Duc, P. Schroeter, and J. Biguen. Spatio-temporal robust motion estimation and segmentation. Lecture Notes in Computer Science, 970:238-245, 1995.

[7] J. Y. A. Wang and E. H. Adelson. Spatio-temporal segmentation of video data. Technical Report 262, MIT Media Lab Vismod, 1994.

[8] F. Moscheni, S. Bhattacharjee and M. Kunt. Spatiotemporal segmentation based on region merging. IEEE TPAMI, 20(9):897-915, September 1998.

[9] Z. Kim and K. Wohn. Spatio-temporal analysis of image sequence: Edge detection and optical flow estimation. Master's thesis, CS Dept., Kaist, 1996.

[10] K. Otsuka, T. Horikoshi, and S. Suzuki. Image velocity estimation from trajectory surface in spatiotemporal space. In CVPR97, pages 200-205, 1997.

[11] S. Peng. Temporal slice analysis of image sequences. In CVPR'91, pages 283-288, 1991.

[12] A. Imiya, K. Kawamoto. Random sampling and voting method for three-dimensional reconstruction. In International Workshop RobVis 2001, Proceedings, volume 1998 of Lecture Notes in Computer Science, pages 193-200. Springer, 2001.

[13] J. Illingworth and J. Kittler. A survey of the Hough transform. Computer Vision, Graphics, and Image Processing, 44(1):87-116, 1988. 
[14] G. Borgefors. Distance transformations in arbitrary dimensions. Computer Vision, Graphics, and Image Processing, 27(3):321-345, 1984.

[15] E. R. White. Assessment of Line-Generalization Algorithms Using Characteristic Points. The American Cartographer 12(1): 17-28, 1985.

[16] D. H. Douglas, T. K. Peucker. Algorithms for the Reduction of the Number of Points Required to Represent a Line or its Caricature. The Canadian Cartographer 10(2): 112-122, 1973.

[17] U. Ramer. An iterative procedure for the polygonal approximation of plane curves. Computer Vision, Graphics, and Image Processing, 1:244-256, 1972

[18] G. Rote. The convergence rate of the Sandwich algorithm for approximating convex functions. Computing, 48:337361, 1992

[19] R. B. McMaster. A statistical analysis of mathematical measures for linear simplification. The American Cartographer, 13:103-116, 1986.

[20] R. Hartley, A. Zisserman. Multiple view geometry in computer vision. Cambridge University Press, 2000

[21] W. H. Press, S. A. Teukolsky, W. T. Vetterling, B. P. Flannery. Numerical recipes in $\mathrm{C}$, second edition. Cambridge University Press, 1993.

[22] M. Sonka, V. Hlavac, and R. Boyle. Image Processing, Analysis and Machine Vision. Chapman and Hall Computing, 1993 